\title{
DETERMINATION OF THE $\pi^{+} \rightarrow \pi^{0}+e^{+}+v$ DECAY RATE
}

\author{
P. Depommier(*), J. Heintze, A. Mukhin ${ }^{(*)}$, C. Rubbia, V. Soergel and K. Winter
}

CERN, Genève

(presented by J. Heintze)

We have measured the rate of the process

$$
\pi^{+} \rightarrow \pi^{0}+e^{+}+v
$$

The branching ratio

$$
\frac{\pi^{+} \rightarrow \pi^{0}+e^{+}+v}{\pi^{+} \rightarrow \mu^{+}+v}
$$

is expected to be of the order of $10^{-9}$ to $10^{-7}$, the conserved vector current theory leading to a well defined value of $\left.(1.06 \pm 0.02) \times 10^{-8} 1\right)$.

The detection of the process is based on a measurement of the energies both of the two $67.5 \mathrm{MeV} \gamma$-rays from the $\pi^{0}$ decay and of the decay positron. The positrons are emitted with an allowed $\beta$-spectrum having the end point at $4.0 \mathrm{MeV}$. Furthermore we require that the $\gamma$-rays and the decay positron are in prompt coincidence, but delayed with respect to the pion stopping in the apparatus.

Fig. 1 shows the counter arrangement. The $70 \mathrm{MeV}$ pion beam of the CERN Synchro-cyclotron passes the input telescope (counters 1 and 2), and stops in the central part of counter 3 . The positron energy is measured in the same counter and the $\gamma$-rays are recorded in counters 5 and 6 . The $\gamma$-detectors are shielded against charged particles by anticoincidence counters 7 and 8.

The recording system connected to the counters is made in such a way that we store as much information as possible with a minimum of electronic preselection. In this way, we get quite clear ideas about the back- ground which could possibly simulate good events. We display the signals of all counters on a fast scope and on a 4-beam scope, whenever a $\gamma \gamma$-coincidence occurs in a time interval of 300 ns around a "stop" in counter 3. A similar method for the detection of rare decay processes has been used, e.g. by Ashkin et al. ${ }^{2)}$.

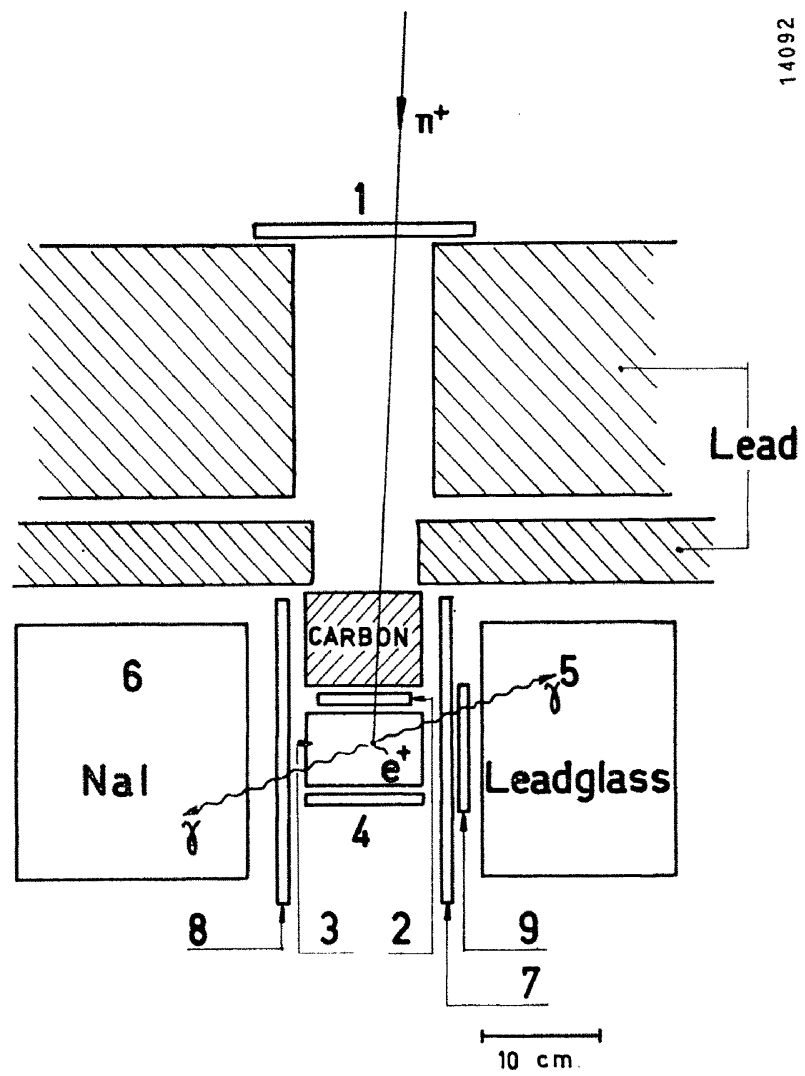

Fig. 1 Counter arrangement. The radiations occuring in the case of a good event are schematically indicated. Counter 9 is used for the detection of the $\pi^{+} \rightarrow e^{+}+\nu+\gamma$ decay which is described in the subsequent paper.

(*) On leave from the Laboratoire de Physique Nucléaire, Université de Grenoble, Grenoble.

(**) On leave from the Joint Institute for Nuclear Research, Dubna. 
Fig. 2 shows the scope displays in the case of a good event. On the fast scope display, the incoming pion gives rise to the signal 1 and to the large pulse from counter 3. The signals can be clearly identified by their size, shape and sign. After some time, (16 ns in this case), the pion stored in counter 3 disintegrates. The decay positron gives the second signal 3 , the amplitude of which is proportional to the positron energy (1.5 MeV in this case). The $\gamma$-rays, which are emitted simultaneously, are represented by standardized time marks 5 and 6 (timing accuracy $\pm 1.3 \mathrm{~ns}$ ). The delays of the pulses are arranged so that the prompt coincidences (13) and (356) appear on the scope as

\section{FAST SCOPE}

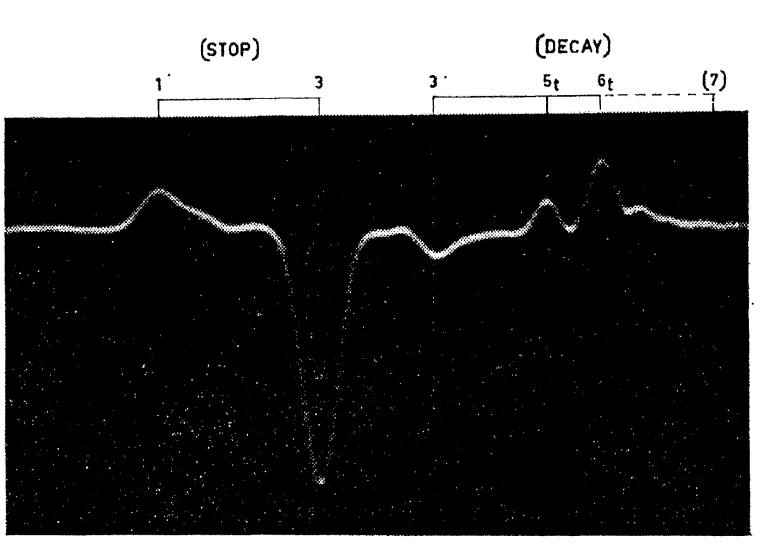

\section{BEAM SCOPE}

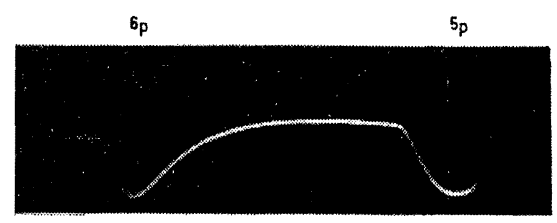

Trace 1

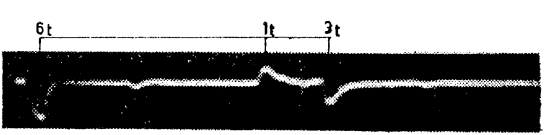

Trace 2

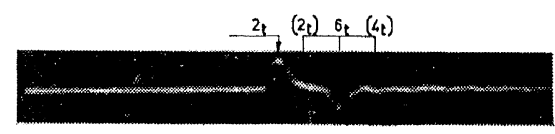

Trace 3

\section{(8)} (9) (

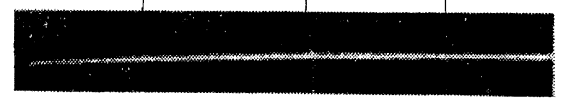

Trace 4

Fig. 2 Scope display for a good event. Above the pulses the numbers of the corresponding counters are indicated (see Fig. 1). The indices $t$ and $p$ mean that the signal contains time or pulse height information only, respectively. Numbers in brackets indicate signals which should be absent in the case of a good $\pi^{+}-\pi^{0}$ event. $T$ is the scope trigger signal coming from the part of the electronics which selects $\pi^{+} \rightarrow e^{+}+v+\gamma$ events. The horizontal brackets on top of the traces indicate the position of signals which aro in prompt coincidence. two groups of separated signals having fixed spacings between the signals in each group. The pulse of counter 7, which is also displayed on the fast scope, is absent in the case of a good event.

The energies of the two $\gamma$-rays are recorded on trace 1 of the 4-beam scope. On trace 2 we put all signals occurring in counters 1 and 3 in a time extending from $4 \mu \mathrm{s}$ before the event to $4 \mu \mathrm{s}$ after. In this way, the " history" of the event is investigated. Trace 3 shows signals from counters 2 and 4 . As a time reference, counter 6 is displayed on traces 2 and 3 in convenient positions. Trace 4 gives the anticoincidence counter 8 and signals connected to the investigation of the radiative $\pi-e$ decay described in the following paper.

During the run, a total of $2.6 \times 10^{10}$ pions were stopped, and 67 patterns were recorded, in which on the fast scope display a (13) coincidence followed by a (356) coincidence was observed, as shown in Fig. 2. Since we must be sure to avoid scanning losses and measuring errors, we restrict the further analysis to patterns in which the 3 (decay) signal comes at least $10 \mathrm{~ns}$ after the 3 (stop) pulse and has a pulse height of at least twice the trace width $(0.7 \mathrm{MeV})$. This leaves 54 events. If we require approximately correct $\gamma$-ray energies (50 $\mathrm{MeV}<E_{6}<100 \mathrm{MeV}, 40 \mathrm{MeV}<E_{5}<110 \mathrm{MeV}$ ), correct positron energy $\left(0.7 \mathrm{MeV}<E_{\beta}<4.5 \mathrm{MeV}\right)$ and the condition that the 3 (decay) pulse has not the signature of a muon [i.e. a given pulse height of 3 (decay) and an additional late decay pulse on trace 2 of the 4-beam scope], we retain 16 events.

Now we have to analyze the background. Let us consider first all the 54 events having a good "fast scope pattern". We made a background subtraction according to the following considerations:

(1) There could be one accidental signal in the decay coincidence (356). The number of background events of this kind is easily deduced from the number of patterns where one signal $(3,5$ or 6$)$ is clearly out of timing. We found 130 events of this type, which leads to 16 events in the "good timing interval". Therefore, $38 \pm 4$ of our events have a real threefold coincidence (356).

(2) Such a real coincidence may be produced either by primary decay products of the stopped $\pi$ or by something else. In the latter case, the stopped pion undergoes $\mu$ decay, but the muon is not seen, for example, because it is hidden in the large pion stop 
pulse. Consequently there must be a corresponding number of patterns where the muon is visible between the stop pulse and the decay pulse in counter 3. 28 events of this kind have been recorded. From the timing conditions and from the analysis of $1000 \pi-\mu$ decays, we know the probability to miss the decay muon and we find that 21 background events from this source have to be subtracted. Therefore we are left with $17 \pm 7$ events from primary pion decays, the big error being due to the background subtraction.

In the same way we can subtract the background from the 16 events selected previously by the criteria specific to the $\pi^{+}-\pi^{0}$ decay. The number of control patterns meeting the corresponding requirements is very small, as expected. We found 4 " out of timing" events (instead of 130) and 2 events with a visible $\mu$ (instead of 28). After the background subtraction, we obtain $14 \pm 2$ events. The agreement with the number obtained before $(17 \pm 7)$, at a high background level, convinces us that we treated the background properly.

The only kind of background not considered up to now is the one produced by decay modes of the pion different from the $\pi^{+}-\pi^{0}$ decay. Here we see the only possibility in radiative $\pi-e$ decays in which $\gamma$-ray and electron go into opposite directions, both having high energies. Such processes are very rare and have not yet been observed. Therefore, we investigated them experimentally, as described in the following paper. However, to give a background event, it is necessary that the electron produces a second energetic $\gamma$-ray in the target counter. From an experimental study using $\mu$-decay electrons, this turned out to be sufficiently improbable.

The conclusion is, therefore, that we have observed $14 \pm 2 \pi^{+}-\pi^{0}$ decay events. This statement is supported by the energy distributions of the decay products in the selected events (Fig. 3a, b).

The probability to detect the $\gamma$-rays was determined with $\pi^{0}$ 's from the reaction $\pi^{-}+p \rightarrow \pi^{0}+n$ occurring at rest in the target counter and by Monte Carlo calculations. Taking into account further reductions of efficiency introduced by energy cuts and the limitations of the observed decay time, we get a value of $(3.2 \pm 0.4) \%$ for the efficiency to detect a $\pi^{+} \rightarrow \pi^{0}$ decay. The branching ratio is then

$$
R=\frac{\pi^{+} \rightarrow \pi^{0}+e^{+}+v}{\pi^{+} \rightarrow \mu^{+}+v}=(1.7 \pm 0.5) \times 10^{-8} .
$$

This is compatible within 1.3 standard errors with the value predicted by the conserved vector current theory.
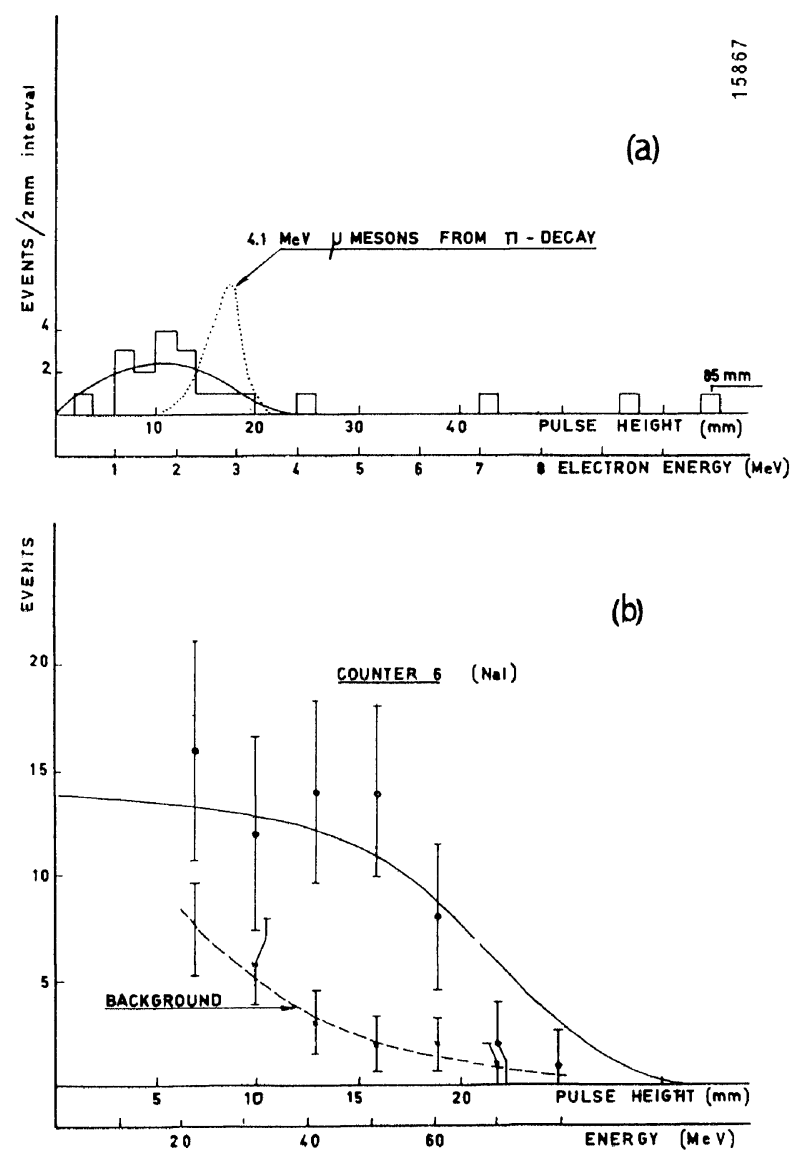

Fig. 3 (a) Spectrum of decay pulses in counter 3. The solid line is an allowed $\beta$ spectrum with $E_{\max }=4.0 \mathrm{MeV}$. Note that the $\mathrm{e}^{+}$annihilation radiation contributes to the measured energy in counter $3(\sim 0.5 \mathrm{MeV}$ on the average). The 3 events above 4.5 $\mathrm{MeV}$ are compatible with the background expected from radiative $\mu$ decay. $\mathrm{A} \mathrm{Na}{ }^{24}$ source has been used to calibrate the pulse height for electrons.

(b) Integral spectrum of the $\mathrm{Nal}$ crystal after background subtraction. The solid line gives the spectrum of Panofsky $\gamma$ rays. The background spectrum is shown separately.

Spectra (a) and (b) contain the good events selected without restrictions on the pulse height in counters 3 and 6 respectively.

\section{LIST OF REFERENCES}

1. S. S. Gershtejn and Ya. B. Zel'dovich, Soviet physics, JETP 2, 576 (1956);

R. P. Feynman and M. Gell-Mann, Phys. Rev. 109, 193 (1958).

G. Da Prato and G. Putzolu, Nuovo Cimento 21, 541 (1961).

2. J. Ashkin, T. Fazzini, G. Fidecaro, N. H. Lipman, A. W. Merrison and H. Paul. Nuovo Cimento 14, 1266 (1959). 


\title{
DISCUSSION
}

Crawford: Trivial question: do you really mean $14 \pm 2$ when the square root of 14 is 3.5 ?

HeInTze: 14 is the number of observed events, and the uncertainty of \pm 2 comes in because of the uncertainty in the number of background counts subtracted. In working out the branching ratio we took of course also the statistical error into account.

BERNSTEIN: Has the uncertainty in the theoretical result to do with the radiative corrections?
HeINTZE: The quoted uncertainty is the combined error of the constants used in the evaluation.

SOERGEL: The biggest contribution comes from the error in the pion lifetime.

BERNARDINI: There is also a contribution from the uncertainty in the mass difference between $\pi^{+}$and $\pi^{0}$.

Heintze: A recent measurement has reduced this uncertainty to a very small amount.

\section{EXPERIMENTAL EVIDENCE FOR STRUCTURE EFFECTS IN THE $\pi^{+} \rightarrow \mathbf{e}^{+}+\boldsymbol{v}+\gamma$ DECAY PROCESS}

\author{
P. Depommier(*), J. Heintze, A. Mukhin ${ }^{(*)}$, C. Rubbia, V. Soergel and K. Winter \\ CERN, Genève
}

(presented by C. Rubbia)

It has been pointed out by several authors ${ }^{1-4)}$ that evidence for pion structure could be obtained from the $\pi^{+} \rightarrow e^{+}+v+\gamma$ decay process, by looking for high energy electron gamma-ray pairs emitted in opposite directions. Experimental evidence for such a process is given in this preliminary report. The experiment has been performed simultaneously with the detection of the $\pi^{+} \rightarrow \pi^{0}+e^{+}+v$ decay mode, described in the preceding paper. In addition to the $\gamma \gamma$ coincidences (from $\pi^{0}$ decay), we recorded, in a different channel of our electronics, $e \gamma$ coincidences. The $\gamma$ ray was detected in the NaI crystal and the decay electron by a coincidence between the large lead glass Čerenkov counter and a $9 \times 10 \mathrm{~cm}^{2}$ plastic scintillator, counter 9 (see Fig. 1 of preceding paper). The events are recorded, as in the $\pi^{+}-\pi^{0}$ experiment, on a fast scope and a 4-beam scope. Fig. 2 of the preceding paper shows also the position of the signals which have to be present in the case of a $\pi^{+} \rightarrow e^{+}+v+\gamma$ event.
Twelve $\pi^{+} \rightarrow e^{+}+v+\gamma$ events have been seen, obeying the proper timing conditions, and with $E_{\gamma}>55 \mathrm{MeV}$ and $E_{e^{+}}>40 \mathrm{MeV}$. The evaluation of the background contribution to these events follows very closely the lines of the preceding paper.

\section{Accidental ey coincidences}

The resolving time of the coincidence circuit is about three times larger than the actual precise timing measured on the film. Therefore, from 3 events out of timing actually observed, we expect $1 \pm 0.5$ events as a background contribution.

2. Radiative $\mu^{+}$decays $\left(\mu^{+} \rightarrow \mathrm{e}^{+}+\nu+\bar{v}+\gamma\right)$ or a real $(e \gamma)$ coincidence accidentally associated with a stopping pion.

Such events will show on the scope display of the target counter, with a known probability, a visible $\mu^{+}$

(*) On leave from the Laboratoire de Physique Nucléaire, Université de Grenoble, Grenoble.

(**) On leave from the Joint Institute for Nuclear Research, Dubna. 